\title{
Work and energy in inertial and non inertial reference frames
}

\author{
Rodolfo A. Diaz; William J. Herrerał Diego A. Manjarrés $\ddagger$ \\ Departamento de Física. Universidad Nacional de Colombia. Bogotá, Colombia.
}

\section{Abstract}

It is usual in introductory courses of mechanics to develop the work and energy formalism from Newton's laws. On the other hand, literature analyzes the way in which forces transform under a change of reference frame. Notwithstanding, no analogous study is done for the way in which work and energy transform under those changes of reference frames. We analyze the behavior of energy and work under such transformations and show explicitly the expected invariance of the formalism under Galilean transformations for one particle and a system of particles. The case of non inertial systems is also analyzed and the fictitious works are characterized. In particular, we show that the total fictitious work in the center of mass system vanishes even if the center of mass defines a non inertial frame. Finally, some subtleties that arise from the formalism are illustrated by examples.

Keywords: Work and energy, fundamental theorem of work and energy, reference frames, galilean transformations.

PACS: $01.55 .+b, 45.20 . \mathrm{Dd}, 45.50 .-\mathrm{j}, 45.20 . \mathrm{dg}$

\section{General formulation}

The behavior of the work and energy formulation under a change of reference frame is not considered in most of the standard literature [1, and when considered, is limited to some specific cases [2]. Recently, the importance of Galilean invariance of the work energy theorem has been highlighted by showing that the assumption of such an invariance implies the impulse theorem 3 . Thus we shall treat the problem in a framework more general than previous approaches: the work energy theorem in inertial and non inertial reference frames for an interacting non isolated system of particles.

Let us assume an inertial reference frame $\Sigma$ and another (inertial or non inertial) reference frame $\Sigma^{\prime}$. The origin of $\Sigma^{\prime}$ moves arbitrarily with respect to $\Sigma$ but there is no relative rotation between them. If $\mathbf{r}$ is the position of a particle in $\Sigma$ then the position $\mathbf{r}^{\prime}$ of the particle in $\Sigma^{\prime}$ reads

$$
\mathbf{r}^{\prime}=\mathbf{r}-\mathbf{R},
$$

where $\mathbf{R}$ is the position of the origin of $\Sigma^{\prime}$ measured by $\Sigma$. For the most general kinematics of such an origin this relation becomes

$$
\mathbf{r}^{\prime}=\mathbf{r}-\mathbf{R}_{0}-\int_{0}^{t} \mathbf{V}\left(t^{\prime}\right) d t^{\prime}
$$

*radiazs@unal.edu.co

†jherreraw@unal.edu.co

‡damanjarrnsg@unal.edu.co with $\mathbf{V}(t)$ denoting the velocity of $\Sigma^{\prime}$ with respect to $\Sigma$,

$$
\mathbf{V}(t)=\mathbf{V}_{0}+\int_{0}^{t} \mathbf{A}\left(t^{\prime}\right) d t^{\prime}
$$

where $\mathbf{R}_{0}, \quad \mathbf{V}_{0}$ are the initial values of $\mathbf{R}$, and V respectively. $\mathbf{A}(t)$ refers to the acceleration of $\Sigma^{\prime}$ as observed by $\Sigma$. We work in a scenario in which time intervals and masses are invariants. By differentiation of Eq. (11) we get

$$
\mathbf{v}^{\prime}=\mathbf{v}-\mathbf{V}(t)
$$

If we start with Newton's second law and use the fact that $\Sigma^{\prime}$ observes a fictitious force on the $j$-th particle of the form $\mathbf{F}_{\text {fict }}=-m_{j} \mathbf{A}(t)$, we get

$$
m_{j} \frac{d \mathbf{v}_{j}^{\prime}}{d t}=\mathbf{F}_{j}-m_{j} \mathbf{A}(t),
$$

$\mathbf{F}_{j}$ denotes the net real force on the $j$-particle i.e. the sum of internal and external forces exerted on it. Taking the dot product with $\mathbf{v}_{j}^{\prime} d t$ (on left) and $d \mathbf{r}_{j}^{\prime}$ (on right), we obtain

$$
m_{j} \mathbf{v}_{j}^{\prime} \cdot d \mathbf{v}_{j}^{\prime}=\left[\mathbf{F}_{j}-m_{j} \mathbf{A}(t)\right] \cdot d \mathbf{r}_{j}^{\prime},
$$

after summing over $j$, it leads to the work and energy theorem in $\Sigma^{\prime}$

$$
d K^{\prime}=d W^{\prime} .
$$

So the theorem of work and energy holds in the non inertial system $\Sigma^{\prime}$ as long as the fictitious forces and their corresponding fictitious works are included in $W^{\prime}$ as expected. Galilean invariance is obtained by using $\mathbf{A}(t)=0$, from which equality still holds and fictitious forces and works dissapear. It is useful to obtain $d K^{\prime}$ and $d W^{\prime}$ in terms of variables measured in the inertial frame $\Sigma$. We do this by using Eqs. (2, 3, 4) and including the fictitious force on the $j$-th particle

$$
\begin{aligned}
d K_{j}^{\prime} & =m_{j} \mathbf{v}_{j}^{\prime} \cdot d \mathbf{v}_{j}^{\prime} \\
& =m_{j}\left[\mathbf{v}_{j}-\mathbf{V}(t)\right] \cdot\left[d \mathbf{v}_{j}-\mathbf{A}(t) d t\right], \\
d W_{j}^{\prime} & =\mathbf{F}_{j}^{\prime} \cdot d \mathbf{r}_{j}^{\prime} \\
& =\left[\mathbf{F}_{j}-m_{j} \mathbf{A}(t)\right] \cdot\left[d \mathbf{r}_{j}-\mathbf{V}(t) d t\right] .
\end{aligned}
$$

Expanding these expressions we find

$$
\begin{aligned}
d K_{j}^{\prime} & =d K_{j}+d Z_{j}, \\
d W_{j}^{\prime} & =d W_{j}+d Z_{j},
\end{aligned}
$$




$$
d Z_{j} \equiv-\mathbf{V}(t) \cdot \mathbf{F}_{j} d t-m_{j}\left[d \mathbf{r}_{j}-\mathbf{V}(t) d t\right] \cdot \mathbf{A}(t) .
$$

From Eqs. 17, 10, 11) and summing over $j$ we get $d K=d W$ as expected. In the galilean case $\mathbf{A}(t)=0$, we find

$$
\begin{aligned}
& d W_{j}^{\prime}=d W_{j}-\mathbf{V} \cdot \mathbf{F}_{j} d t=d W_{j}-\mathbf{V} \cdot d \mathbf{P}_{j}, \\
& d W^{\prime}=d W-\mathbf{V} \cdot \mathbf{F} d t=d W-\mathbf{V} \cdot d \mathbf{P},
\end{aligned}
$$

where $d \mathbf{P}_{j}$ denotes the differential of linear momentum associated with the $j$-particle. The second of these equations is obtained just summing the first equation over $j$. It is easy to check that $d \mathbf{P}_{j}$ is the same for any inertial reference frame, but if $\Sigma^{\prime}$ is non inertial we should take into account that $d \mathbf{P}_{j}$ is always measured by $\Sigma$ as can be seen in (12).

When $\Sigma^{\prime}$ is non inertial, it is customary to separate $d W^{\prime}$ in the work coming from real forces and the work coming from fictitious forces. The work associated with fictitious forces can be easily visualized in Eq. (9) and we write

$$
\begin{aligned}
d W^{\prime} & =d W_{\text {real }}^{\prime}+d W_{\text {fict }}, \\
d W_{\text {fict }} & =-\mathbf{A}(t) \cdot \sum_{j=1}^{n} m_{j}\left[d \mathbf{r}_{j}-\mathbf{V}(t) d t\right],
\end{aligned}
$$

and Eq. (7) can be rewritten in the following way

$$
d W_{\text {real }}^{\prime}+d W_{\text {fict }}=d K^{\prime},
$$

which is similar in structure to the corresponding equation for forces. An interesting case arises when $\Sigma^{\prime}$ is attached to the center of mass of the system. In that case, the total differential of fictitious work reads

$$
d W_{\text {fict }}^{C M}=-\mathbf{A}_{C}(t) \cdot \sum_{j} m_{j} d \mathbf{r}_{j}^{\prime},
$$

with $\mathbf{A}_{C}$ denoting the acceleration of the center of mass. Since the masses $m_{j}$ are constant and using the total mass $M$ we get

$$
d W_{\text {fict }}^{C M}=-M \mathbf{A}_{C}(t) \cdot d\left(\frac{\sum_{j} m_{j} \mathbf{r}_{j}^{\prime}}{M}\right)=0 .
$$

The term in parenthesis vanishes because it represents the position of the center of mass around the center of mass itself. So the total fictitious work in the center of mass system vanishes even if such a system is non inertial. Notice however that the total fictitious force does not necessarily vanish, neither the fictitious work done over a specific particle.

It is worth emphasizing that the reference frame attached to the center of mass posseses some particular properties: (a) the relation between angular momentum and torque $d \mathbf{L}_{C M} / d t=\tau_{C M}$ holds even if the CM system is non inertial, (b) the fictitious forces do not contribute to the total external torque 4, (c) the fictitious forces do not contribute to the total work as proved above. We point out however, that this situation is different when the $\mathrm{CM}$ frame rotates with respect to an inertial frame [4].

It is clear that the case of one particle arises by supressing the $j$ index and only external forces appear on the particle. The case in which $\Sigma^{\prime}$ is inertial (galilean transformation) appears when $\mathbf{A}=0$, and in that case we see that (a) the fictitious forces and works dissappear, (b) the fundamental theorem holds in the same way as appears in $\Sigma$, that is $d W_{\text {real }}^{\prime}=d K^{\prime}$. It is worth remarking that when $\Sigma^{\prime}$ is attached to the center of mass the relation $d W_{\text {real }}^{\prime}=d K^{\prime}$ also holds even if $\Sigma^{\prime}$ is non inertial.

\section{Examples}

There are other subtleties on this formulation that could be enlightened by some examples

Example 1: Consider a block pushed across a frictionless table by a constant force $\mathbf{F}$ through a distance $L$ starting at rest (as observed by $\Sigma$ ). So $\Delta K=W$ implies $m v^{2} / 2=$ $F L$ and the time taken is $t=m v / F$. But the table is in the dining car of a train travelling with constant speed $V$ (measured by $\Sigma^{\prime}$ ) in the direction of $\mathbf{F}$. From the point of view of $\Sigma^{\prime}$, the work done on the block and its change in the kinetic energy read

$$
\begin{aligned}
W^{\prime} & =F(L+V t)=F(L+m v V / F)=F L+m v V \\
\Delta K^{\prime} & =\frac{1}{2} m(V+v)^{2}-\frac{1}{2} m V^{2}=\frac{1}{2} m v^{2}+m v V
\end{aligned}
$$

which shows explicitly that $\Delta K=W$ implies $\Delta K^{\prime}=W^{\prime}$. The reader can check that we find the same result by using Eq. (13).

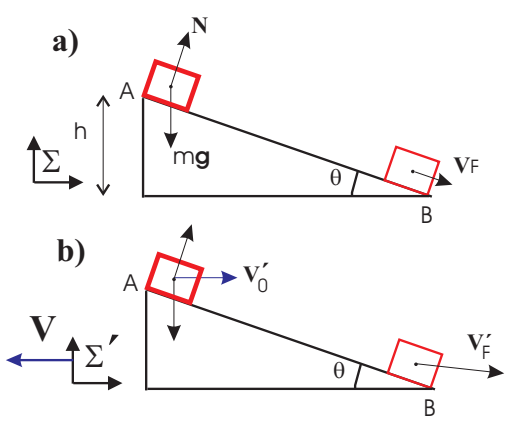

Figure 1: Illustration of example 2. Force diagrams of a block over a wedge, (a) with respect to $\Sigma$, (b) with respect to $\Sigma^{\prime} . \mathbf{N}, m \mathbf{g}$ denote the normal and gravitational forces respectively.

Example 2: Consider a block of mass $m$ sliding over a frictionless wedge. In the $\Sigma$ frame, the block is at height $h$ and at rest when $t=0$ (see Fig. 1). The block will be our physical system of interest. Assume that the reference frame $\Sigma^{\prime}$ is another inertial system traveling with velocity $\mathbf{V}=-u \mathbf{i}$ toward left (see Fig. 1). We intend to calculate the work on the block and its final speed in the $\Sigma^{\prime}$ frame. For this, we apply Eq. (13) in a finite form

$$
\begin{aligned}
W^{\prime} & =W-\mathbf{V} \cdot \mathbf{I}=m g h-(-u \mathbf{i}) \cdot m\left[\mathbf{v}_{f}-\mathbf{v}_{0}\right] \\
& =m g h+m u \mathbf{i} \cdot \mathbf{v}_{f}
\end{aligned}
$$


where $\mathbf{I}$ denotes impulse. By arguments of energy in $\Sigma$ we see that $v_{F}=\sqrt{2 g h}$ so that

$$
W^{\prime}=m g h+m u \sqrt{2 g h} \cos \theta .
$$

It can be checked by calculating explicitly the work due to each force, that the extra term in $W^{\prime}$ owes to the fact that the normal force contributes to the work in $\Sigma^{\prime}$. It is because the trajectory of the block in $\Sigma^{\prime}$ is not perpendicular to the normal force. Since the theorem of work and energy is valid in $\Sigma^{\prime}$ and all forces are conservative, we can use the conservation of mechanical energy applied to points $A$ and $B$ to get

$$
\frac{1}{2} m u^{2}+m g h+m u \sqrt{2 g h} \cos \theta=\frac{1}{2} m v_{F}^{\prime 2},
$$

and solving for $v_{F}^{\prime}$ we find

$$
v_{F}^{\prime}=\left(u^{2}+2 g h+2 u \sqrt{2 g h} \cos \theta\right)^{1 / 2} .
$$

It is easy to check the consistency of Eq. (18) since $\mathbf{v}_{0}^{\prime}=u \mathbf{i}$ and $\mathbf{v}_{F}^{\prime}$ can be obtained by taking into account that $\mathbf{v}_{F}^{\prime}=$ $\mathbf{v}_{F}+u \mathbf{i}$ and using $v_{F}=\sqrt{2 g h}$ from which we obtain Eq. (18). One of the main features illustrated by this problem is that the work done by a force depends on the reference frame, and consequently its associated potential energy (if any). In the case in which both systems are inertial, forces are the same in $\Sigma$ and $\Sigma^{\prime}$. However, works done by each force can change because the trajectories are different in each system. For this particular problem, from the point of view of $\Sigma^{\prime}$ the weight works in the same way as observed by $\Sigma$, but the normal force does work which is opposite to the observations in $\Sigma$. What really matters is that the work and energy theorem holds in both $*$. Finally, it is worth saying that although the constraint force in this example produces a real work on the block in $\Sigma^{\prime}$, it is a consequence of the motion in time of the wedge as observed by $\Sigma^{\prime}$, therefore it does not produce a virtual work in the sense of D'Alembert principle [5], hence such a principle holds in $\Sigma^{\prime}$ as well.

Example 3: Consider a ball that with respect to $\Sigma$ is in vertical free fall in a uniform gravitational field $\mathbf{g}$ starting at rest. Assume that $\Sigma^{\prime}$ is non inertial with acceleration $\mathbf{A}=-a \mathbf{k}$ and initial velocity $\mathbf{V}_{0}=-V_{0} \mathbf{k}$ with respect to $\Sigma$ (see Fig. 2). The total work on the ball from $A$ to $B$ measured by $\Sigma^{\prime}$ involves real and fictitious forces and can be obtained by integrating Eq. (9)

$$
\begin{aligned}
& W^{\prime}=m(a-g) \mathbf{k} \cdot\left\{\int_{\mathbf{r}_{A}}^{\mathbf{r}_{B}} d \mathbf{r}+\int_{0}^{T}\left(V_{0}+a t\right) \mathbf{k} d t\right\}, \\
& W^{\prime}=m(g-a)\left(z-V_{0} T-a T^{2} / 2\right),
\end{aligned}
$$

*If we applied naively the conservation of energy by assigning the traditional potential energies for $m \mathbf{g}$ and $\mathbf{N}$ ( $m g h$ and zero respectively), we would obtain the mechanical energies $E_{A}=m g h+$ $(1 / 2) m u^{2}$ and $E_{B}=(1 / 2) m v_{F}^{\prime 2}$. Using conservation of mechanical energy and applying Eq. (18) we obtain that such an equality can only holds for the particular cases $u=0$ or $\theta=0$. Such a contradiction comes from an incorrect use of the potential energies when changing the reference frame. Recalling that the potential energy associated with a constant force $\mathbf{F}$ (in $\Sigma$ ) is $-\mathbf{F} \cdot \mathbf{r}+$ const, and taking into account that $\mathbf{N}$ and $m \mathbf{g}$ are constant (in both frames), then suitable potential energies for both forces in $\Sigma^{\prime}$ can be constructed by using potential energies of the form $-\mathbf{F} \cdot \mathbf{r}^{\prime}+$ const.

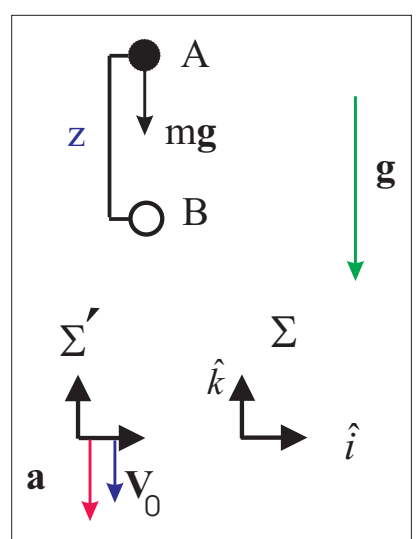

Figure 2: Illustration of example 3. A ball in free fall with respect to $\Sigma . \Sigma^{\prime}$ has a uniform acceleration with respect to $\Sigma$.

where $z$ is the distance covered by the ball from $A$ to $B$ as observed by $\Sigma$, and $T$ the time in covering such a distance. When $g=a$ then $W^{\prime}=0$. This fact can be seen from the equivalence principle, since in the case in which $\mathbf{a}=\mathbf{g}$ the gravitational equivalent field associated with the non inertial system cancels out the external uniform field, so the force measured by such a system (and so the work) vanishes. Finally, the reader can check that the fundamental theorem of work and energy holds in $\Sigma^{\prime}$ for this case, as long as we use the work $W^{\prime}$ which includes the fictitious work.

\section{Conclusions}

We have examined the behavior of the work and energy formulation for a system of particles under a change of reference frame. We show explicitly the galilean covariance of the work and energy theorem and show the way in which such a theorem behaves in a non inertial frame. It is worth pointing out that the form of the theorem is preserved when going to a non inertial traslational frame as long as the fictitious works are included. In addition, we found that when the reference frame is attached to the center of mass, the total fictitious work is always null such that the work and energy theorem is held without the inclusion of fictitious works even if such a frame is non inertial. Finally, we illustrate the fact that after a change of reference frame, the work done for each forces also changes (even if the transformation is galilean). In consequence, the corresponding potential energies should be changed when they exist. In particular, we show that normal forces could produce work in some inertial reference frames.

\section{ACKNOWLEDGMENTS}

We acknowledge the useful suggestions of two anonymous referees. We also thank División de Investigación de Bogotá (DIB) from Universidad Nacional de Colombia, for its financial support. 


\section{A Suggestions for readers}

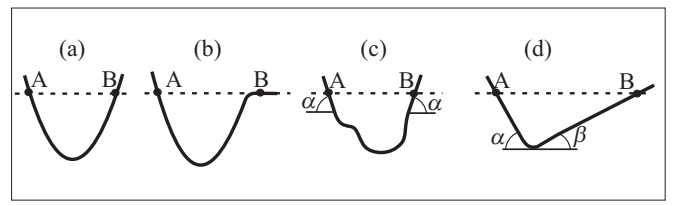

Figure 3: Some configurations of a ball sliding over a frictionless surface. The ball travels from point $A$ to point $B$, and such points are fixed with respect to the surface.

For the reader to assimilate the principal features exposed, we propose the following situations and questions,

- Let us consider two balls of masses $m_{1}, m_{2}$ in free fall in a uniform gravitational field with respect to a frame attached to the ground $\Sigma$. The mass $m_{1}$ starts at rest from a height $h$ while the mass $m_{2}$ is thrown upward from the ground on the same vertical with an initial velocity $\mathbf{v}_{0}$. We ask the reader to show explicitly that the total work measured by the center of mass system $\Sigma_{C}$ is null and therefore the total kinetic energy remains constant. The fact that the fictitious work is zero is a general property for $\Sigma_{C}$ as stated in Eq. (15), while the fact that the work done for real forces vanishes is a particular characteristic of this problem.

- As it has been noted previously, when we change of reference frame, an amount of work associated with forces that do not do work in the initial frame can appear. In the galilean case this amount of work is easily evaluated from the net impulse. Consider a block of mass $m$ sliding over different arrangements as depicted in Fig. 3. For all cases, the block is at rest in the position $A$ with respect to the $\Sigma$ frame, and travels from $A$ to $B$. Another inertial frame $\Sigma^{\prime}$ is introduced, which moves with speed $V$ to the left. Without making any explicit calculations. For which cases does the normal force perform net work between the points $A$ and $B$ in $\Sigma^{\prime}$ ?.

- For our second example, sketch adequate potential energies for the block in both $\Sigma$ and $\Sigma^{\prime}$. Does the potential energy exhibit the same behaviour in both frames?, Why or Why not?. Further, calculate explicitly the net work on the block observed by $\Sigma^{\prime}$. This is another way of checking that Eq. (16) holds.

- Imagine the very typical problem of a block sliding on a frictionless surface, starting at a height $h$ and ending at $h=0$. We inmediately put $m g h=m v^{2} / 2$. However, the conservation of mechanical energy requires to use the potential energy associated with the net force on the block, while in this case we are using the potential energy associated with an applied force which clearly differs from the net force, under what circumstances is the typical result correct?

\section{References}

[1] D. Kleppner, R. Kolenkow An introduction to mechanics (Mc Graw-Hill Publishing Company,1973); M. Alonso, E. Finn Fundamental University Physics, Vol I, Mechanics (Addison-Wesley Publishing Co.,1967).

[2] A. Mallinckrodt, H. Leff All about work, Am. J. Phys. 60 356-365 (1992); I. Galili, D. Kaplan Extending the application of the relativity principle: Some pedadogical advantages, Am. J. Phys 65, 328-335 (1997); S. Madhu Rao The kinematical roots of dynamics Am. J. Phys. 68, 329-333 (2000); B. J. Tefft, J. A. Tefft Galilean Relativity and the work-kinetic energy theorem, The Physics Teacher 45, 218-220 (2007).

[3] M. Camarca, A. Bonanno, P. Sapia, Revisiting workenergy theorem's implications, Eur. J. Phys. 28, 11811187 (2007).

[4] Rodolfo A. Diaz, William J. Herrera, On the transformation of torques between the laboratory and center of mass reference frames, Revista Mexicana de Física E 51 (2) 112-115 (2005).

[5] H. Goldstein, C. Poole, J. Safko, Classical Mechanics, 3rd Ed. (Addison Wesley, 2002). 